\title{
Semisynthesis of Esters of Fraxinellone C4/10-Oxime and Their Pesticidal Activities
}

Qin $\mathrm{Li}^{\dagger}$, Xiaobo Huang ${ }^{\dagger}$, Shaochen $\mathrm{Li}^{\dagger}$, Jingchun $\mathrm{Ma}^{\dagger}$, Min $\mathrm{Lv}^{*}{ }^{\dagger}$, and Hui $\mathrm{Xu}^{*, \dagger, \star}$

${ }^{\dagger}$ College of Plant Protection, Northwest A\&F University, Yangling 712100, Shaanxi

Province, China.

${ }^{\ddagger}$ Shaanxi Key Laboratory of Natural Products \& Chemical Biology, Northwest A\&F

University, Yangling 712100, Shaanxi Province, China.

* Author to whom correspondence should be addressed [(H.X.) telephone:

+86(0)29-87091952; fax: +86(0)29-87091952; e-mail: orgxuhui@nwsuaf.edu.cn;

lvmin@nwsuaf.edu.cn (M. L.)].

Data for 7e: Yield: $92 \%$, white solid, m.p. $172-174{ }^{\circ} \mathrm{C} ;\left[\alpha{ }^{20}{ }_{\mathrm{D}}=5\left(\right.\right.$ c 3.3, $\left.\mathrm{CHCl}_{3}\right)$; IR $\mathrm{cm}^{-1}$ (KBr): 3149, 3066, 2930, 1761, 1649, 1266, 1064, 931, 765, 709; ${ }^{1} \mathrm{H}$ NMR (500 $\left.\mathrm{MHz}, \mathrm{CDCl}_{3}\right) \delta:$ 8.08-8.10 (m, 2H, Ar-H), $7.61(\mathrm{t}, J=8.0 \mathrm{~Hz}, 1 \mathrm{H}, \mathrm{Ar}-\mathrm{H}), 7.48-7.51$ (m, 4H, H-2', 5', Ar-H), 6.37 (s, 1H, H-4'), 5.06 (s, 1H, H-8), 3.31-3.37 (m, 1H, H-5), 2.65-2.73 (m, 1H, H-5), 2.50 (s, 3H, H-10), 1.97-2.01 (m, 1H, H-6), 1.80-1.86 (m, 1H, H-6), 0.96 (s, 3H, H-11); HRMS (ESI): Calcd for $\mathrm{C}_{21} \mathrm{H}_{20} \mathrm{O}_{5} \mathrm{~N}\left([\mathrm{M}+\mathrm{H}]^{+}\right), 366.1336$; Found, 366.1336.

Data for 7f: Yield: 84\%, white solid, m.p. $201-203{ }^{\circ} \mathrm{C}$; $\left[\alpha{ }^{20}{ }_{\mathrm{D}}=1\right.$ (c 2.4, $\left.\mathrm{CHCl}_{3}\right)$; IR $\mathrm{cm}^{-1}$ (KBr): $3114,3056,2929,1756,1646,1272,1094,932,877,735 ;{ }^{1} \mathrm{H}$ NMR (500 $\left.\mathrm{MHz} \mathrm{CDCl}_{3}\right) \delta: 7.87-7.89$ (m, 2H, Ar-H), 7.51 (s, 1H, H-2'), 7.48 (s, 1H, H-5'), 7.42 (d, $J=7.5 \mathrm{~Hz}, 1 \mathrm{H}, \mathrm{Ar}-\mathrm{H}), 7.36$ (t, $J=7.5 \mathrm{~Hz}, 1 \mathrm{H}, \mathrm{Ar}-\mathrm{H}), 6.36$ (s, 1H, H-4'), 5.06 (s, 1H, H-8), 3.31-3.36 (m, 1H, H-5), 2.65-2.73 (m, 1H, H-5), 2.50 (s, 3H, H-10), 2.43 (s, $\left.3 \mathrm{H},-\mathrm{CH}_{3}\right), 1.98-2.01$ (m, 1H, H-6), 1.80-1.86 (m, 1H, H-6), 0.96 (s, 3H, H-11); HRMS (ESI): Calcd for $\mathrm{C}_{22} \mathrm{H}_{22} \mathrm{O}_{5} \mathrm{~N}\left([\mathrm{M}+\mathrm{H}]^{+}\right), 380.1492$; Found, 380.1485 .

Data for 7g: Yield: 90\%, white solid, m.p. $140-142{ }^{\circ} \mathrm{C} ;[\alpha]^{20}{ }_{\mathrm{D}}=0.2\left(\right.$ c 2.6, $\left.\mathrm{CHCl}_{3}\right)$; IR cm ${ }^{-1}$ (KBr): 3129, 3050, 2930, 1755, 1648, 1250, 1056, 930, 837; ${ }^{1} \mathrm{H}$ NMR (500 
$\left.\mathrm{MHz}, \mathrm{CDCl}_{3}\right) \delta: 7.97$ (d, J=8.0 Hz, 2H, Ar-H), 7.51 (s, 1H, H-2'), 7.48 (s, 1H, H-5'), 7.28 (d, $J=8.0 \mathrm{~Hz}, 2 \mathrm{H}, \mathrm{Ar}-\mathrm{H}), 6.36$ (s, 1H, H-4'), 5.06 (s, 1H, H-8), 3.31-3.35 (m, 1H, H-5), 2.64-2.72 (m, 1H, H-5), 2.50 (s, 3H, H-10), 2.44 (s, 3H, - $\mathrm{CH}_{3}$ ), 1.97-2.00 (m, 1H, H-6), 1.79-1.86 (m, 1H, H-6), 0.96 (s, 3H, H-11); HRMS (ESI): Calcd for $\mathrm{C}_{22} \mathrm{H}_{22} \mathrm{O}_{5} \mathrm{~N}\left([\mathrm{M}+\mathrm{H}]^{+}\right)$, 380.1492; Found, 380.1491.

Data for 7h: Yield: $88 \%$, white solid, m.p. $182-184{ }^{\circ} \mathrm{C}$; $\left[\alpha{ }^{20}{ }_{\mathrm{D}}=-3\left(\right.\right.$ c $\left.2.8, \mathrm{CHCl}_{3}\right)$; IR $\mathrm{cm}^{-1}$ (KBr): 3125, 3078, 2934, 1736, 1606, 1258, 1069, 928, 763; ${ }^{1} \mathrm{H}$ NMR (500 MHz, $\left.\mathrm{CDCl}_{3}\right) \delta: 8.04(\mathrm{~d}, J=9.0 \mathrm{~Hz}, 2 \mathrm{H}, \mathrm{Ar}-\mathrm{H}), 7.47-7.51$ (m, 2H, H-2', 5'), 6.96 (d, $J=9.0$ $\mathrm{Hz}, 2 \mathrm{H}, \mathrm{Ar}-\mathrm{H}), 6.37$ (s, 1H, H-4'), 5.06 (s, 1H, H-8), 3.88 (s, 3H, - $\left.\mathrm{OCH}_{3}\right), 3.30-3.35$ (m, 1H, H-5), 2.64-2.71 (m, 1H, H-5), 2.50 (s, 3H, H-10), 1.96-2.00 (m, 1H, H-6), 1.79-1.85 (m, 1H, H-6), 0.95 (s, 3H, H-11); HRMS (ESI): Calcd for $\mathrm{C}_{22} \mathrm{H}_{22} \mathrm{O}_{6} \mathrm{~N}$ $\left([\mathrm{M}+\mathrm{H}]^{+}\right), 396.1442 ;$ Found, 396.1440.

Data for 7i: Yield: $87 \%$, white solid, m.p. $194-196{ }^{\circ} \mathrm{C}$; $\left[\alpha{ }^{20}{ }_{\mathrm{D}}=1\left(\right.\right.$ c 2.2, $\left.\mathrm{CHCl}_{3}\right)$; IR $\mathrm{cm}^{-1}$ (KBr): 3145, 3035, 2925, 1762, 1650, 1250, 1075, 929, 901, 739; ${ }^{1} \mathrm{H}$ NMR (500 $\left.\mathrm{MHz} \mathrm{CDCl}_{3}\right) \delta: 8.04$ (s, 1H, Ar-H), 7.98 (d, $\left.J=8.0 \mathrm{~Hz}, 1 \mathrm{H}, \mathrm{Ar}-\mathrm{H}\right), 7.59$ (d, $J=9.0$ Hz, 1H, Ar-H), 7.51 (s, 1H, H-2'), 7.48 (s, 1H, H-5'), 7.43 (t, J= 7.5 Hz, 1H, Ar-H), 6.37 (s, 1H, H-4'), 5.07 (s, 1H, H-8), 3.30-3.35 (m, 1H, H-5), 2.66-2.74 (m, 1H, H-5), 2.49 (s, 3H, H-10), 1.99-2.03 (m, 1H, H-6), 1.80-1.87 (m, 1H, H-6), 0.96 (s, 3H, $\mathrm{H}-11)$; HRMS (ESI): Calcd for $\mathrm{C}_{21} \mathrm{H}_{19} \mathrm{O}_{5} \mathrm{NCl}\left([\mathrm{M}+\mathrm{H}]^{+}\right)$, 400.0946; Found, 400.0940. Data for 7j: Yield: $83 \%$, white solid, m.p. $130-132{ }^{\circ} \mathrm{C}$; $\left[\alpha{ }^{20}{ }_{\mathrm{D}}=9\left(\right.\right.$ c 3.2, $\left.\mathrm{CHCl}_{3}\right)$; IR $\mathrm{cm}^{-1}$ (KBr): 3139, 3047, 2929, 1762, 1652, 1234, 1029, 957, 747; ${ }^{1} \mathrm{H}$ NMR (500 MHz, $\left.\mathrm{CDCl}_{3}\right) \delta: 7.85(\mathrm{~d}, J=7.5 \mathrm{~Hz}, 1 \mathrm{H}, \mathrm{Ar}-\mathrm{H}), 7.47-7.50$ (m, 4H, H-2', 5', Ar-H), 7.36-7.39 $(\mathrm{m}, 1 \mathrm{H}, \operatorname{Ar}-\mathrm{H}), 6.35$ (s, 1H, H-4'), 5.05 (s, 1H, H-8), 3.29-3.33 (m, 1H, H-5), 2.58-2.66 (m, 1H, H-5), 2.48 (s, 3H, H-10), 1.94-1.97 (m, 1H, H-6), 1.77-1.84 (m, 1H, H-6), 0.94 (s, 3H, H-11); HRMS (ESI): Calcd for $\mathrm{C}_{21} \mathrm{H}_{19} \mathrm{O}_{5} \mathrm{NCl}\left([\mathrm{M}+\mathrm{H}]^{+}\right), 400.0946$; Found, 400.0944.

Data for 7k: Yield: $91 \%$, white solid, m.p. $187-189{ }^{\circ} \mathrm{C}$; $[\alpha]{ }_{\mathrm{D}}^{20}=7 \times 10^{-2}$ (c 2.0, $\left.\mathrm{CHCl}_{3}\right)$; IR cm ${ }^{-1}(\mathrm{KBr}): 3148,3081,2931,1756,1650,1238,1055,925,768 ;{ }^{1} \mathrm{H}$ NMR (500 MHz, $\left.\mathrm{CDCl}_{3}\right) \delta: 8.34(\mathrm{~d}, J=9.0 \mathrm{~Hz}, 2 \mathrm{H}, \mathrm{Ar}-\mathrm{H}), 8.25(\mathrm{~d}, J=8.5 \mathrm{~Hz}, 2 \mathrm{H}$, Ar-H), 7.48-7.52 (s, 2H, H-2', 5'), 6.37 (s, 1H, H-4'), 5.08 (s, 1H, H-8), 3.30-3.35 (m, 1H, H-5), 2.68-2.76 (m, 1H, H-5), 2.50 (s, 3H, H-10), 1.99-2.03 (m, 1H, H-6), 1.82-1.89 (m, 1H, H-6), 0.97 (s, 3H, H-11); HRMS (ESI): Calcd for $\mathrm{C}_{21} \mathrm{H}_{19} \mathrm{O}_{7} \mathrm{~N}_{2}$ $\left([\mathrm{M}+\mathrm{H}]^{+}\right), 411.1187$; Found, 411.1187.

Data for 7l: Yield: $81 \%$, white solid, m.p. $188-190{ }^{\circ} \mathrm{C} ;[\alpha]^{20}=12\left(c 2.4, \mathrm{CHCl}_{3}\right)$; IR 
$\mathrm{cm}^{-1}$ (KBr): 3107, 3028, 2929, 1756, 1647, 1221, 1101, 962, 805, 715; ${ }^{1} \mathrm{H}$ NMR (500 $\left.\mathrm{MHz}, \mathrm{CDCl}_{3}\right) \delta: 8.87(\mathrm{~s}, 1 \mathrm{H}, \mathrm{Ar}-\mathrm{H}), 8.48(\mathrm{~d}, J=8.5 \mathrm{~Hz}, 1 \mathrm{H}, \mathrm{Ar}-\mathrm{H}), 8.43$ (d, $J=8.0$ $\mathrm{Hz}, 1 \mathrm{H}, \mathrm{Ar}-\mathrm{H}), 7.72$ (t, $J=8.0 \mathrm{~Hz}, 1 \mathrm{H}, \mathrm{Ar}-\mathrm{H}), 7.52$ (s, 1H, H-2'), 7.49 (s, 1H, H-5'), 6.37 (s, 1H, H-4'), 5.08 (s, 1H, H-8), 3.33-3.37 (m, 1H, H-5), 2.70-2.78 (m, 1H, H-5), 2.50 (s, 3H, H-10), 2.02-2.05 (m, 1H, H-6), 1.83-1.89 (m, 1H, H-6), 0.98 (s, 3H, H-11); HRMS (ESI): Calcd for $\mathrm{C}_{21} \mathrm{H}_{19} \mathrm{O}_{7} \mathrm{~N}_{2}\left([\mathrm{M}+\mathrm{H}]^{+}\right)$, 411.1187; Found, 411.1185. Data for $7 \boldsymbol{m}$ : Yield: $91 \%$, white solid, m.p. $163-165{ }^{\circ} \mathrm{C}$; $\left[\alpha{ }^{20}{ }_{\mathrm{D}}=3\left(\mathrm{c} 3.6, \mathrm{CHCl}_{3}\right)\right.$; IR $\mathrm{cm}^{-1}$ (KBr): 3148, 3069, 2932, 1759, 1651, 1224, 1047, 928, 837; ${ }^{1} \mathrm{H}$ NMR (500 MHz, $\left.\mathrm{CDCl}_{3}\right) \delta:$ 8.10-8.13 (m, 2H, Ar-H), 7.48-7.51 (m, 2H, H-2', 5'), 7.15-7.19 (m, 2H, Ar-H), 6.36 (s, 1H, H-4'), 5.06 (s, 1H, H-8), 3.29-3.34 (m, 1H, H-5), 2.64-2.72 (m, 1H, H-5), 2.49 (s, 3H, H-10), 1.97-2.01 (m, 1H, H-6), 1.80-1.86 (m, 1H, H-6), 0.96 (s, 3H, $\mathrm{H}-11)$; HRMS (ESI): Calcd for $\mathrm{C}_{21} \mathrm{H}_{19} \mathrm{O}_{5} \mathrm{NF}\left([\mathrm{M}+\mathrm{H}]^{+}\right)$, 384.1242; Found, 384.1241.

Data for $7 \boldsymbol{n}(\boldsymbol{E} / \boldsymbol{Z}=3: 2)$ : Yield: $89 \%$, white solid, m.p. $140-142{ }^{\circ} \mathrm{C} ;\left[\alpha{ }^{20}{ }_{\mathrm{D}}=26(c) 3.6\right.$, $\mathrm{CHCl}_{3}$ ); IR cm ${ }^{-1}$ (KBr): 3149, 3041, 2980, 1735, 1654, 1224, 1097, 925, 900, 759; ${ }^{1} \mathrm{H}$ NMR (500 MHz, $\left.\mathrm{CDCl}_{3}\right) \delta:$ 7.45-7.56 (m, 4.4H, H-2', 5', Ar-H), 7.27-7.30 (m, 0.8H, Ar-H), 7.01-7.05 (m, 0.8H, Ar-H), 6.34 (s, 0.4H, H-4'), 6.32 (s, 0.6H, H-4'), 5.02 (s, 0.4H, H-8), 4.97 (s, 0.6H, H-8), 4.29 (s, 1.2H, $-\mathrm{CH}_{2-}$ ), 3.82 (s, 0.8H, $-\mathrm{CH}_{2-}$ ), 3.04-3.09 (m, 0.4H, H-5), 2.78-2.83 (m, 0.6H, H-5), 2.41-2.48 (m, 0.4H, H-5), 2.40 (s, 1.2H, H-10), 2.36 (s, 1.8H, H-10), 2.19-2.26 (m, 0.6H, H-5), 1.89-1.93 (m, 0.4H, H-6), 1.70-1.80 (m, 1.0H, H-6), 1.59-1.66 (m, 0.6H, H-6), 0.91 (s, 1.2H, H-11), 0.85 (s, 1.8H, H-11); HRMS (ESI): Calcd for $\mathrm{C}_{22} \mathrm{H}_{21} \mathrm{O}_{5} \mathrm{NF}\left([\mathrm{M}+\mathrm{H}]^{+}\right), 398.1398$; Found, 398.1396.

Data for 8f: Yield: $87 \%$, white solid, m.p. $115-117^{\circ} \mathrm{C}$; $[\alpha]^{20}{ }_{\mathrm{D}}=-24\left(c 2.1, \mathrm{CHCl}_{3}\right)$; IR $\mathrm{cm}^{-1}$ (KBr): 3146, 3061, 2943, 1745, 1651, 1271, 1086, 922, 882, 735; ${ }^{1} \mathrm{H}$ NMR (500 $\left.\mathrm{MHz}, \mathrm{CDCl}_{3}\right) \delta: 9.40$ (s, 1H, H-10), 7.91-7.93 (m, 2H, Ar-H), 7.51 (s, 1H, H-2'), 7.48 (s, 1H, H-5'), 7.42 (d, J=7.5 Hz, 1H, Ar-H), 7.36 (t, $J=7.5 \mathrm{~Hz}, 1 \mathrm{H}, \mathrm{Ar}-\mathrm{H}), 6.38$ (s, 1H, H-4'), 5.06 (s, 1H, H-8), 2.93 (dd, $J=20.0,6.0 \mathrm{~Hz}, 1 \mathrm{H}, \mathrm{H}-4), 2.60-2.67$ (m, 1H, H-4), 2.44 (s, 3H, - $\mathrm{CH}_{3}$ ), 1.99-2.04 (m, 1H, H-5), 1.88-1.92 (m, 1H, H-6), 1.76-1.85 (m, 1H, H-5), 1.54-1.60 (m, 1H, H-6), 0.99 (s, 3H, H-11); HRMS (ESI): Calcd for $\mathrm{C}_{22} \mathrm{H}_{22} \mathrm{O}_{5} \mathrm{~N}\left([\mathrm{M}+\mathrm{H}]^{+}\right), 380.1492$; Found, 380.1492 .

Data for 8i: Yield: 95\%, white solid, m.p. $153-155^{\circ} \mathrm{C}$; $[\alpha]^{20}{ }_{\mathrm{D}}=-15\left(\right.$ c 2.6, $\left.\mathrm{CHCl}_{3}\right)$; IR $\mathrm{cm}^{-1}$ (KBr): $3128,3028,2945,1748,1653,1254,1081,964,875,739 ;{ }^{1} \mathrm{H}$ NMR (500 $\left.\mathrm{MHz}, \mathrm{CDCl}_{3}\right) \delta: 9.39$ (s, 1H, H-10), 8.09 (s, 1H, Ar-H), 7.99 (d, J=8.0 Hz, 1H, Ar-H), 
$7.58(\mathrm{~d}, J=7.5 \mathrm{~Hz}, 1 \mathrm{H}, \mathrm{Ar}-\mathrm{H}), 7.51\left(\mathrm{~s}, 1 \mathrm{H}, \mathrm{H}-2^{\prime}\right), 7.48$ (s, 1H, H-5'), 7.42 (t, $J=8.0$ Hz, 1H, Ar-H), 6.37 (s, 1H, H-4'), 5.06 (s, 1H, H-8), 2.91 (dd, $J=20.0,6.0 \mathrm{~Hz}, 1 \mathrm{H}$, H-4), 2.59-2.66 (m, 1H, H-4), 2.00-2.03 (m, 1H, H-5), 1.89-1.91 (m, 1H, H-6), 1.77-1.86 (m, 1H, H-5), 1.56-1.60 (m, 1H, H-6), 0.99 (s, 3H, H-11); HRMS (ESI): Calcd for $\mathrm{C}_{21} \mathrm{H}_{19} \mathrm{O}_{5} \mathrm{NCl}\left([\mathrm{M}+\mathrm{H}]^{+}\right), 400.0946$; Found, 400.0944.

Data for $8 \boldsymbol{k}$ : Yield: $82 \%$, white solid, m.p. $111-113{ }^{\circ} \mathrm{C} ;\left[\alpha{ }^{20}{ }_{\mathrm{D}}=-20\left(\right.\right.$ c 2.0, $\left.\mathrm{CHCl}_{3}\right)$; IR $\mathrm{cm}^{-1}$ (KBr): 3110, 3076, 2925, 1758, 1654, 1262, 1078, 964, 761; ${ }^{1} \mathrm{H}$ NMR (500 MHz, $\left.\mathrm{CDCl}_{3}\right) \delta: 9.43$ (s, 1H, H-10), 8.33 (d, $\left.J=8.0 \mathrm{~Hz}, 2 \mathrm{H}, \mathrm{Ar}-\mathrm{H}\right), 8.28$ (d, $J=8.0 \mathrm{~Hz}, 2 \mathrm{H}$, Ar-H), 7.48-7.51 (m, 2H, H-2', 5'), 6.37 (s, 1H, H-4'), 5.07 (s, 1H, H-8), 2.91 (dd, $J=$ 20.0, 6.5 Hz, 1H, H-4), 2.59-2.66 (m, 1H, H-4), 2.01-2.05 (m, 1H, H-5), 1.90-1.93 (m, 1H, H-6), 1.77-1.87 (m, 1H, H-5), 1.56-1.61 (m, 1H, H-6), 1.00 (s, 3H, H-11); HRMS (ESI): Calcd for $\mathrm{C}_{21} \mathrm{H}_{19} \mathrm{O}_{7} \mathrm{~N}_{2}\left([\mathrm{M}+\mathrm{H}]^{+}\right), 411.1187$; Found, 411.1186 . 